FORMATION Formation emploi

Revue française de sciences sociales

108 | octobre-décembre 2009

Les processus de professionnalisation

\title{
Une professionnalisation problématique : les experts judiciaires interprètes-traducteurs
}

Problematische Professionalisierung: Dolmetscher- und Übersetzer bei Gericht*

Problematic skills training: Expert legal translators/interpreters

Una profesionalizacion problematica : los expertos judiiales interpretes-

traductores

Keltoume Larchet et Jérôme Pélisse

\section{OpenEdition}

Journals

Édition électronique

URL : http://journals.openedition.org/formationemploi/2051

DOI : 10.4000/formationemploi.2051

ISSN : 2107-0946

Éditeur

La Documentation française

Édition imprimée

Date de publication : 1 décembre 2009

Pagination : $9-24$

ISSN : 0759-6340

Référence électronique

Keltoume Larchet et Jérôme Pélisse, «Une professionnalisation problématique : les experts judiciaires interprètes-traducteurs », Formation emploi [En ligne], 108 | octobre-décembre 2009, mis en ligne le 01 janvier 2012, consulté le 30 octobre 2020. URL : http://journals.openedition.org/formationemploi/ 2051 ; DOI : https://doi.org/10.4000/formationemploi.2051 


\section{DOSSIER}

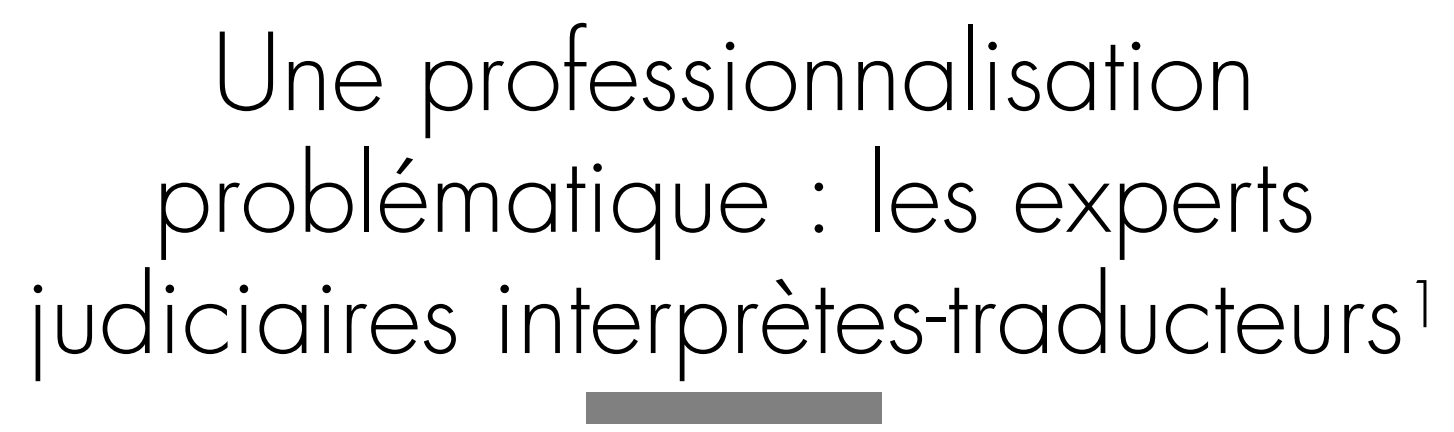

Keltoume Larchet, Jérôme Pélisse*

Les experts interprètes-traducteurs sont à la fois experts judiciaires nommés par des juges, prestataires de services assermentés en langues pour une clientèle privée, garants d'un droit fondamental, et parfois professionnels d'un autre domaine. Cette diversité d'appartenances engendre une professionnalisation structurellement problématique.

Dans un contexte d'européanisation et d'internationalisation des espaces juridiques et judiciaires, mais aussi de complexification croissante des règles définissant les conditions de séjour des étrangers, l'activité de traduction et d'interprétariat pour le compte de la justice ouvre un certain nombre d'enjeux. L'un d'entre eux concerne la manière d'exercer professionnellement cette activité indispensable au fonctionnement de la justice, thème jamais étudié en France, parmi les nombreux travaux portant sur les professions juridiques ou judiciaires ${ }^{2}$.

${ }^{1}$ L'article s'appuie sur une recherche collective, dirigée par J. Pélisse, dans le cadre d'une convention avec la Mission de recherche Droit et Justice. Cette recherche, engagée fin 2007 et achevée fin 2009, regroupait K. Larchet, C. Protais (doctorante à l'EHESS - École des hautes études en sciences sociales) et E. Charrier (professeur associé à l'université Paris Dauphine). Voir www.hicjacet.org/1252/index.html pour plus de détails.

${ }^{2}$ Voir par exemple les travaux de L. Karpik (1996) sur les avocats, V. Roussel (2007) sur les magistrats, A. Mathieu-Fritz (2005) sur les huissiers de justice, ou L. Dumoulin (2007) sur les experts judiciaires. Quant à la profession d'interprète ou de traducteur, il existe quelques travaux sur les traducteurs littéraires (N. Heinich,
Keltoume Larchet est doctorante en sociologie à I'IDHE Cachan (Institutions et Dynamiques Historiques de l'Économie, UMR ENS-CNRS), et monitrice à l'université de Reims, depuis octobre 2008. Elle étudie le marché professionnel des interprètes traducteurs (sous la direction de P.P. Zalio etJ. Pélisse).

Jérôme Pélisse est maître de conférences en sociologie à l'université de Reims, chercheur à l'IDHE Cachan. Parmi ses publications les plus récentes: "Judiciarisation ou juridicisation? Usages et réappropriations du droit dans les conflits du travail », Politix nº 86, 2009 ; avec R. Melot, "Prendre la mesure du droit : enjeux de l'observation statistique pour la sociologie juridique », Droit et Société, $n^{\circ}$ 69-70, 2008 ; avec Beroud S., Denis J.-M., Desage G., Giraud B., La lutte continue? Les conflits du

\section{........}

1984 ou G. Sapiro, 2008 par exemple) mais aucun ne s'intéresse à l'activité de traduction assermentée (traduction certifiée conforme au regard du droit) ou pour le compte de la justice (lors de procès, d'instruction judiciaire, de garde à vue). 
travail dans la France contemporaine, Coll. "Raison d'agir », Le Croquant, Bellecombes en Bauge, 2008. Les auteurs remercient la Mission de recherche Droit et Justice qui a financé l'étude dont est issu en partie cet article, ainsi que L. Dumoulin, les trois relecteurs anonymes et l'équipe de rédaction de la revue Formation Emploi pour leurs commentaires et suggestions.

Il est en effet de rigueur, en France, que les juges puisent dans des listes d'experts judiciaires les techniciens et les auxiliaires leur permettant d'éclairer, par un savoir de spécialiste, tel ou tel point sur les affaires qu'ils sont en charge de juger. Ces experts, qui ne sont pas pour autant des professionnels du droit puisqu'ils ne sont nommés comme experts judiciaires qu'en raison de leur professionnalité non juridique, constituent un groupe intéressant à étudier du point de vue de la sociologie des professions. Contrepartie d'un «professionnalisme orthodoxe » exigé par les magistrats, une professionnalisation ambiguë, implicite et non reconnue, a ainsi pu être mise en évidence par L. Dumoulin (2007) à partir d'une analyse simultanément historique, juridique et sociologique. Elle y montre comment les autorités judiciaires ont constamment refusé officiellement de faire des experts judiciaires une profession, tout en contribuant à les professionnaliser de manière souterraine par leurs pratiques, et comment ces derniers, pour acquérir un professionnalisme leur permettant d'être nommés et reconnus, ont eux-mêmes endossé un modèle du « bon expert» largement construit par les magistrats. Celuici représente un professionnel inachevé, à la fois expert de son domaine et disponible pour l'institution judiciaire, vivant de son activité et non de ses prestations pour la justice, malgré la particularité des activités et l'expérience exigée de lui en la matière.

L'objet de cet article est de reprendre ce cadre d'analyse et de le mettre à l'épreuve d'une spécialité originale, celle des experts interprètes-traducteurs, peu investiguée par L. Dumoulin. Bien qu'existant comme une catégorie ancienne des listes d'experts judiciaires éditées par les Cours d'Appel ${ }^{3}$, les experts

\footnotetext{
${ }^{3}$ Pour une perspective générale et historique, voir F. Chauvaud et L. Dumoulin (2003). Même si nous n'avons pu retrouver la date à partir de laquelle les interprètes-traducteurs ont constitué une catégorie d'expert judiciaire, on peut mentionner en effet que, .../...
}

interprètes-traducteurs constituent en effet un groupe particulier, hétérogène et marginal, au sein de celui des experts judiciaires, toujours soupçonné de ne pas être constitué de "véritables» experts. Eclatés et hétérogènes, peu unifiés et peu représentés par des instances les regroupant, les experts interprètestraducteurs constituent un ensemble de professionnels à la professionnalisation problématique, malgré l'importance du titre d'expert judiciaire pour la régulation du marché privé de la traduction et de l'interprétariat.

Dans quelle mesure observe-t-on une professionnalisation des experts interprètes-traducteurs au sens d'un "processus selon lequel un corps de métier tend à s'organiser sur le modèle des professions établies» (Chapoulie, 1973) ? En quoi peut-il être qualifié de problématique ? C'est dans cette optique que seront abordés les formations expertales, les instances représentatives, le sentiment d'appartenance, appréhendés comme autant d'indices de professionnalisation inséparables de l'existence d'une entité professionnelle. Il ne s'agit pas, toutefois, d'en rester à une conception de la professionnalisation comme processus linéaire qui placerait les interprètes-traducteurs en quelque sorte « en retard» vis-à-vis d'autres experts judiciaires. En prenant en compte, au sein de cette dynamique incertaine, les enjeux et les comportements d'autres acteurs que sont les magistrats, la Chancellerie, les autres experts judiciaires et la clientèle privée des interprètestraducteurs, on pourra en effet approfondir la nature problématique de cette professionnalisation et les enjeux qui la traversent.

Articulant diverses données et méthodologies, l'article examine donc tout d'abord la marginalisation et la dévalorisation de ces experts interprètestraducteurs, avant d'analyser un ensemble d'indices néanmoins fragiles et ambivalents témoignant d'un processus de professionnalisation. Ce mouvement de professionnalisation n'en demeure pas moins semé d'obstacles, liés aux clivages qui structurent ce groupe et au rôle que joue le titre d'expert judiciaire sur le marché privé de la traduction assermentée mais

.../... dès 1911, certains se sont regroupés dans une association spécifique, la Compagnie des experts traducteurs-interprètes, CETI. 


\section{Encadré 1 \\ Données et méthodologies}

Quatre types de matériaux et de traitements sont au fondement de cette recherche, qui décline une étude simultanément individuelle - Keltoume Larchet débute ainsi une thèse sur le sujet - et collective. Dirigée par Jérôme Pélisse, la recherche collective porte sur une sociographie des experts judiciaires dans le domaine économique, psychiatrique et des langues, dans le cadre d'une convention avec la Mission de recherche Droit et Justice.

Des questionnaires postaux ont ainsi été envoyés à tous les experts psychiatres et experts économistes, et à un peu moins de la moitié des experts interprètes-traducteurs en France (soit 1000 questionnaires pour ces derniers), connus via les listes d'experts que tient chacune des 34 cours d'appels françaises. L'échantillon a été construit aléatoirement, en tenant compte du nombre d'experts inscrits par juridiction ; la représentativité des répondants a pu être analysée au regard de quelques critères (sexe, âge, ancienneté, origine géographique) grâce aux informations contenues dans les listes. Le taux de réponse varie en effet selon les juridictions autour d'une moyenne de 14,5\%, comparable à celui des autres spécialités d'expertise étudiées. Au final, malgré une sous-représentation des experts en début d'activité layant une faible ancienneté, ou en situation probatoire $\left({ }^{*}\right)$, qui peut s'expliquer par leur moindre légitimité à exposer des opinions sur leur nouveau statut et activités, l'échantillon apparaît globalement représentatif de l'ensemble des experts interprètes-traducteurs.

Des interrogations sur la trajectoire, le statut, le rapport à l'institution judiciaire et l'activité des experts ont été complétées par trois autres types de données, recueillies à partir de méthodologies plus qualitatives: entretiens auprès d'une quinzaine d'experts interprètes-traducteurs et de cinq magistrats (notamment en charge du service des expertises) tout d'abord ; observations d'une douzaine d'audiences et de cycles de formation, ensuite; analyse de dossiers d'experts anonymisés dans deux cours d'appel, de rapport et de textes législatifs ou réglementaires concernant l'expertise de justice, enfin.

$\left(^{*}\right)$ : C'est-à-dire ayant moins de deux ans d'ancienneté. En effet, une première inscription sur les listes permet d'accéder à un statut dit d'expert probatoire, puisqu'il nécessite un réexamen du dossier au bout de deux ans pour demeurer sur la liste en qualité d'expert.

aussi à la double posture adoptée par les autorités judiciaires à leur égard.

\section{LES EXPERTS INTERPRÈTES- TRADUCTEURS, UN GROUPE MARGINAL ET MARGINALISÉ}

De loin les plus nombreux parmi les experts judiciaires, les interprètes-traducteurs constituent pourtant un groupe marginal et marginalisé au sein de ce groupe «professionnel». Les guillemets sont en effet de rigueur pour évoquer cette activité d'expertise de justice et le statut qui lui est associé.

\section{Un groupe « professionnel »?}

Toutes disciplines confondues (celles-ci allant de l'architecture à la médecine, en passant par l'art), l'expertise de justice tire son fondement de sa dimension accessoire. C'est sur la base d'une expérience avérée et d'une activité en lien avec la spécialité expertale que se justifie l'inscription sur les listes d'experts judiciaires, qui délimitent autant qu'elles constituent ce groupe «professionnel ». Au-delà de conditions d'âge, d'absence de casier judiciaire, de localisation géographique ou de numerus clausus $^{4}$,

\footnotetext{
${ }^{4}$ Bien qu'elle soit implicite et variable, il existe en effet une logique tendant à limiter le nombre d'experts dans une spécialité et une cour d'appel données, renforcée par la loi de 2004 (voir encadré 2).
} 
les critères présidant à l'attribution du titre d'expert judiciaire se déclinent principalement autour des compétences avérées dans le domaine concerné, d'où l'appellation « d'expert ».

\section{Encadré 2}

\section{Le cadre juridico- institutionnel de l'expertise judiciaire et les modifications de 2004}

Le cadre institutionnel du recours à l'expertise de justice est issu du 19e siècle et a été codifié pour l'essentiel en deux temps, dans les années 1930 puis en 1971, avant les modifications apportées par une loi et un décret en 2004. Technicien, auxiliaire du juge et prestataire de service, l'expert judiciaire s'inscrit au cœur du modèle décisionniste de l'expertise : il est étroitement contrôlé par le magistrat qui le nomme ponctuellement pour telle ou telle affaire requérant, selon lui, un avis autorisé auquel il n'est pas tenu. La Chancellerie a ainsi toujours refusé une professionnalisation de cette activité. La réforme de 2004 ne change pas cette construction mais modifie en pratique les conditions d'obtention et de maintien du statut d'expert. Cette loi implique en effet une réinscription quinquennale /l'inscription était auparavant définitive, jusqu'à «l'honorariat » pour les experts de plus de 65 ans), visant à actualiser le contenu des listes parmi lesquelles vont puiser les magistrats. Ces listes par Cour d'appel sont ainsi établies par des commissions de magistrats intégrant également, autre nouveauté depuis 2004, des experts euxmêmes. Pour mettre en œuvre la réinscription quinquennale, les cours d'appels ont choisi d'opérer par tranche: un cinquième des experts sont réinscrits ou non chaque année depuis 2004. L'objectif d'un « nettoyage » des listes, c'est-à-dire de l'éviction d'experts n'ayant (plus) aucune activité ni liens avec l'institution judiciaire, a en effet justifié quasi explicitement cette mesure. Ainsi, lors de l'enquête postale (réalisée début 2008), seuls trois cinquième des experts avaient été soumis à la procédure de réinscription.
Une candidature à l'inscription sur les listes d'experts judiciaires en médecine ne pourra donc être reçue si elle n'émane pas d'un médecin. C'est en cela que, comme le souligne L. Dumoulin (2007), on peut considérer les experts judiciaires comme un groupe professionnel, ou du moins comme un groupe potentiellement engagé dans un processus de professionnalisation. Toutefois, il en va différemment chez les experts interprètes-traducteurs. Rien de systématique en effet à ce que ce dernier soit interprète et/ou traducteur, l'activité professionnelle principale d'un expert interprète-traducteur pouvant être sans lien avec le domaine linguistique. Un premier principe au fondement du statut d'expert est ici rompu, si l'on suit l'insistance de P. Lascoumes (2002) sur «la source de normativité interne permettant de définir l'expert comme celui qui détient un savoir particulier dans un milieu professionnel $»^{5}$.

\section{Des «professionnels» et des «non-professionnels»}

Une première et singulière distinction sépare ainsi « experts interprètes-traducteurs professionnels » (ceux dont l'activité professionnelle principale est également la traduction et l'interprétariat) et « experts interprètes traducteurs non professionnels »- cette terminologie ne renvoyant aucunement à une moindre qualification des seconds. C'est en effet sur la base de cette distinction que se jouent certains des enjeux relatifs à la professionnalisation problématique de ce groupe. De ce point de vue, la répartition est bien plus équilibrée que ne le laissaient penser, au détriment des experts dit professionnels, les experts judiciaires ayant des fonctions de représentation de leurs pairs que nous avions rencontrés pour préparer l'envoi de notre questionnaire. De plus, parmi les nonprofessionnels, le poids des enseignants et des formateurs en langue est également à noter, contrastant avec la très forte hétérogénéité annoncée.

Ce résultat peut toutefois être nuancé si on fait l'hypothèse que le segment le plus mobilisé et le plus «professionnel» des experts a probablement

\footnotetext{
${ }^{5}$ L'expert étant alors désigné comme «un spécialiste reconnu parmi les autres professionnels de sa spécialité » dont la compétence est induite par " une longue pratique ».
} 
Tableau 1

Activité principale des experts interprètes-traducteurs

\begin{tabular}{|l|c|c|}
\hline & Nombre & Fréquence \\
\hline Interprètes - traducteurs (experts interprètes-traducteurs professionnels) & 66 & $45,5 \%$ \\
\hline $\begin{array}{l}\text { Non interprètes - traducteurs (experts interprètes-traducteurs non professionnels), } \\
\text { dont : }\end{array}$ & 79 & $54,5 \%$ \\
\hline Enseignement et formation en langue & 30 & $38,0 \%$ \\
\hline Retraité & 7 & $8,9 \%$ \\
\hline Enseignement supérieur / recherche & 6 & $7,6 \%$ \\
\hline Social & 5 & $6,3 \%$ \\
\hline Immobilier & 4 & $5,0 \%$ \\
\hline Tourisme / hôtellerie & 4 & $5,0 \%$ \\
\hline Autres (dont 6 non réponses) & 23 & $29,0 \%$ \\
\hline Ensemble & 145 & $100,0 \%$ \\
\hline
\end{tabular}

Source : enquête des auteurs (2008).

répondu davantage au questionnaire ${ }^{6}$. Il n'en reste pas moins que, par rapport aux autres spécialités où les experts «non professionnels» (i.e. ayant une activité principale différente de celle pour laquelle ils sont experts judiciaires) représentent une part peu importante, et le plus souvent nulle ${ }^{7}$, les interprètestraducteurs connaissent un clivage particulier. Car même si la représentation d'un groupe complètement atomisé peut être battue en brèche, ce clivage partage bien le groupe en deux ensembles numériquement important, aux intérêts, activités, rapports à l'activité expertale et à l'institution judiciaire a priori différents (voir infra).

\section{Des conditions d'activité difficiles}

D'autres caractéristiques, qu'elles soient sociodémographiques ou liées aux types d'activités pour lesquels ils sont nommés par les juges, sont autant d'indices de la position marginale et dévalorisée de

${ }^{6}$ Si l'on ne peut contrôler ce biais, on peut toutefois le relativiser : s'il existait massivement, il est peu probable que les structures d'âge, de sexe ou de localisation géographique auraient été les mêmes dans la population de départ et dans l'échantillon des répondants.

${ }^{7} \mathrm{C}$ 'est le cas pour les experts comptables et financiers et les experts psychiatres notamment. Mais on peut imaginer un expert en philatélie dont l'activité principale n'a pas de lien avec cette discipline. ces experts. En effet, les caractéristiques sociales des interprètes-traducteurs tranchent singulièrement avec celles des autres experts judiciaires. Largement féminisés $(71 \%$ de femmes, à contraster avec les $18 \%$ d'expertes psychiatres et les $9 \%$ d'expertes comptables), les interprètes-traducteurs sont aussi les plus jeunes et ont le moins d'anciens parmi eux. Leur âge moyen est de 50 ans (57 ans chez les psychiatres et chez les économistes) et leur ancienneté moyenne s'élève à moins de dix ans ${ }^{8}$. Surtout, il $\mathrm{y}$ a beaucoup plus d'experts probatoires parmi les interprètestraducteurs que parmi les autres spécialités (26\% contre $14 \%$ pour les psychiatres et $10 \%$ chez les économistes), ce qui témoigne d'un renouvellement et d'une expansion récente de leur nombre. Si ces traits distinctifs (âge, sexe, ancienneté) ne constituent pas des éléments stigmatisants dans l'absolu, c'est leur agencement qui contribue à dépeindre une représentation dévalorisée de ces experts par rapport aux autres spécialités d'expertise, investies par des hommes, âgés et expérimentés, ayant les traits attendus de « véritables » professionnels.

${ }^{8}$ Il s'agit ici de données nationales fondées sur l'exploitation des listes d'experts et non des résultats à l'enquête par questionnaire, néanmoins comparables. 
Cette marginalité et cette dévalorisation du statut et de l'activité des experts interprètes-traducteurs s'illustrent également par la nature d'une partie de leur «clientèle», la difficulté des conditions de travail qu'ils rencontrent ou les tarifs des missions qu'ils effectuent. Appelés en comparution immédiate, par exemple pour des affaires de petite délinquance ou de sans-papiers, les experts interprètestraducteurs ne sont pas les mieux situés dans la hiérarchie des usagers de la justice. Contrairement aux autres experts judiciaires, qui se voient confier des missions pour un temps plus ou moins long et possèdent souvent une capacité à en négocier les délais, les interprètes doivent aussi faire preuve d'une disponibilité temporelle très élevée. Il n'est pas rare qu'ils soient appelés au milieu de la nuit pour se rendre dans un commissariat parfois loin de leur domicile. Concernant les tarifs, la dévalorisation est encore de mise : ce sont de loin les plus bas de tous ceux que perçoivent les experts judiciaires. D'après l'article R 122 du Code de Procédure Pénale - qui constitue la base légale et pratique des rémunérations des experts interprètes-traducteurs -, les tarifs s'élevaient, jusqu'à récemment, à $11,13 €$ pour une page traduite de 250 mots et de 13 à $15 €$ pour une heure d'interprétariat. Ils ont toutefois été doublés fin 2008 pour atteindre $25 €$ par page de traduction et par heure d'interprétariat ${ }^{9}$. Certes, les plaintes récurrentes des experts sur cette question concernent toutes les spécialités : les experts économistes et les psychiatres aussi se plaignent, dans notre questionnaire, du niveau auquel sont rémunérées leurs missions pour le compte de la justice. Mais cette question prend une acuité particulière pour les experts interprètes-traducteurs : plus des trois quart d'entre eux déclaraient ainsi rencontrer, en janvier 2008, ces deux «problèmes majeurs » que sont le niveau des honoraires $(78 \%)$ et surtout celui des délais et retards de paiement (80\%). Enfin, $75 \%$ des répondants aux questionnaires ne sont pas vraiment ou pas du tout d'accord avec l'idée selon laquelle la rémunération de leur activité d'expertise tient compte à sa juste valeur de la difficulté de la prestation, du service rendu à la société et des béné-

9 Avec des majorations pour les prestations réalisées de nuit ou pendant le week-end, qui n'existaient pas jusqu'alors. fices qu'ils en tirent dans leurs pratiques professionnelles et leurs relations sociales.

\section{Une activité singulière et dévalorisée}

Les interprètes-traducteurs n'effectuent pas, en effet, d'interventions sous «forme d'observations, d'investigations, de diagnostic ou de conseils » pour le compte de l'institution judiciaire, conformément à la définition des activités des experts judiciaires que propose L. Dumoulin (op. cit., 2007, p. 16). La spécialité linguistique induit une absence de rapport d'expertise : l'interprétariat se réalise en instantané, et la traduction ne produit pas un document distinct $\mathrm{du}$ support d'origine, sauf cas très exceptionnel. Aucun «art de la pioche $»^{10}$ ne peut être repéré du côté des magistrats quant au produit de l'expertise menée par des interprètes-traducteurs. Il n'y a donc pas de relation magistrat/expert interprète-traducteur spécifique, comme c'est le cas pour d'autres spécialités : leurs interactions sont limitées, sinon inexistantes. Les magistrats n'ont en quelque sorte pas à choisir d'expert interprète-traducteur dans la mesure où les prestations de chacun apparaissent comme transposables quel que soit l'expert mandaté. De fait, le présupposé, largement partagé parmi nos interlocuteurs magistrats ou experts judiciaires d'autres spécialités, suivant lequel le domaine linguistique est exempt de toute ambivalence et qu'il revêt une dimension purement technique où aucun jugement ni aucune « prise » n'intervient ${ }^{11}$, accentue leur isolement. "Ce qu'on demande, c'est que les gens traduisent à peu près ce que le prévenu va dire et c'est souvent des choses très simples. (...) Ce n'est pas un travail très complexe en matière de statut des étrangers ou de comparution immédiate par exemple » explique un magistrat en charge du service des expertises dans une grande cour d'appel.

\footnotetext{
${ }^{10}$ Par cette expression, L. Dumoulin (op. cit., p. 133-136) évoque les manières dont les magistrats puisent, " piochent » et se servent des rapports d'expertise pour prendre leur décision. Les interprètes sont considérés comme n'apportant aucune «plus-value » au magistrat qui attend de l'expertise un éclairage l'aidant à prendre à sa décision.

${ }^{11}$ Complémentaire à l'art de la pioche des magistrats, « l'art de la prise » sur les objets et situations qualifierait en effet le travail d'expertise, selon Dumoulin, qui s'appuie ici sur la perspective développée par C. Bessy et F. Chateaureynaud (1995).
} 
Encadré 3

\section{Des interprètes transparents?}

Lors d'observations d'audiences à la cour d'appel de Paris, plusieurs dimensions témoignent de l'invisibilité du travail de l'interprète, alors même que ce travail d'intermédiation entre le prévenu, la Cour et le public est essentiel. Or, le débit de parole du juge ou du procureur a systématiquement le même rythme qu'un interprète soit présent ou non la présence de ce dernier en est comme annihilée, impression renforcée par le fait que l'interprète chuchote la plupart du temps à destination du prévenu, puisque le volume de propos à traduire vers le prévenu est plus important dans ce sens que dans l'autre. À plusieurs reprises lors de nos observations, la seule référence à l'interprète qui a été faite par le juge a été de vérifier sa présence au début de l'audience.

Cette vision omet pourtant les particularités et les ambiguïtés linguistiques tout autant qu'elle occulte le caractère performatif du droit qui réside dans sa capacité à produire des effets de droits par leur simple énonciation ${ }^{12}$. Comme l'explique P. Bourdieu (1982), «le discours juridique est une parole créatrice, qui fait exister ce qu'elle énonce», ce que l'idée de "pouvoir des mots sous-jacent au droit», avancée par M. Harvey (2005), illustre également : "le droit ne vise [en effet] pas du tout l'univocité. " Dès lors, la traduction juridique ne doit pas tenter de les atténuer ou de s'en accommoder mais plutôt de les intégrer et d'en jouer au service du support visé et ainsi reproduire les «effets de sens multiples ». La difficulté réside alors dans la nécessité de retranscrire l'ambiguïté d'un discours juridique dans une autre langue. La traduction est "une affaire de choix»

\footnotetext{
${ }^{12}$ Que ce soit pour un texte légal ou de « simples » propos tenus dans une salle d'audience, aux mots est assortie une portée de droit : pour évoquer un dérapage récent, comparaît par exemple dans un procès un prévenu et non un coupable. L'effet performatif du droit se manifeste dans le fait que certaines réalités existent par leur énonciation même en droit - le fait de se marier, de reconnaître un enfant ou de jurer de dire la vérité dans un tribunal par exemple.
}

ajoute Harvey. Rien de moins évident en effet que de traduire en langue étrangère des termes aussi essentiels que « droit», « loi » ou «bon père de famille» qui sont autant de concepts soumis à l'interprétation que de manières de qualifier juridiquement des faits, lourdes de conséquences dans le cadre d'un procès. Le terme même de «magistrat », par exemple, ne peut se traduire simplement du français au tchèque, souligne J. Radimsky (2004), qui indique «qu'à moins que ce terme ne soit déterminé par un complément prépositionnel («du siège » ou «du parquet »), il pourra correspondre au terme "soudce" ("le juge »), ou bien au terme "státní zástupce" ("le procureur »)». De même, "le terme tchèque "trestný in" équivaut en français aux termes "délité" ou "crime" qui diffèrent en plusieurs points - par exemple quant à la juridiction compétente ou à la gravité de la peine dont l'infraction en question peut être sanctionnée ». En ce sens, "traduire un terme juridique dans une langue étrangère ne veut dire rien de moins que transposer un système juridique dans un autre » ajoute Radimsky ${ }^{13}$.

\section{Luttes de juridiction et marginalisation}

Ces diverses dimensions propres aux experts interprètes-traducteurs, les marginalisant et les dévalorisant, mettent en question leur légitimité par rapport au reste des experts judiciaires. Que ce soit pour souligner la nécessité de leur réserver un traitement différencié ou pour délégitimer leur posture d'expert, nos interlocuteurs soulignent très souvent ces particularités : «ils [experts interprètes-traducteurs] n'ont pas les mêmes contraintes que les autres en matière d'indépendance. Le principe du contradictoire n'a pas la même teneur pour eux (...) à tel point que la Chancellerie avait envisagé une liste séparée» explique un magistrat, tandis qu'une greffière indique que "les relations avec les compagnies d'experts sont bonnes, le cas des interprètestraducteurs est cependant particulier. Il y a des difficultés avec eux ». Quant aux experts judiciaires non spécialistes en langues également rencontrés dans cette enquête (comptables, psychiatres, autres), ils ont presque toujours souligné les différences, sinon

${ }^{13}$ Sur ces problèmes et difficultés de la traduction juridique qui en font rien moins qu'une opération technique et neutre, voir aussi F. Terré (1986) et J.-C. Gemar (1987). 
l'incongruité, de les considérer au même titre que les autres spécialités dans une étude comparative. Car si "leur présence sur les listes est peut être une bonne chose pour qu'ils soient reconnus, elle n'est pas forcément justifiée » explique par exemple le rédacteur-adjoint d'une revue spécialisée sur l'expertise judicaire.

Peut-on y voir l'illustration de ce qu'Andrew Abbott appelle des conflits de juridiction ${ }^{14}$ ? Ceux-ci pourraient d'ailleurs être menés aussi bien par les magistrats à l'égard des experts, qu'entre spécialités d'expertise - les représentants des experts judiciaires à la tête des compagnies locales ou nationales (jamais interprètes-traducteurs) tentant de cloisonner l'accès au titre d'expert ou, du moins, d'entretenir l'idée que les interprètes-traducteurs ne méritent pas vraiment le titre. Cette démarcation constituerait alors une différenciation symbolique que le reste du corps expertal utiliserait pour justifier de sa position, sinon revendiquer une professionnalisation que l'exemple des interprètes-traducteurs pourrait menacer face aux magistrats et à la Chancellerie. Les interprètes-traducteurs ont d'ailleurs conscience - au point souvent de l'intérioriser - d'être considérés comme des «sous-experts judiciaires». "Que voulez-vous raconter sur les experts interprètestraducteurs? Non! Si vous voulez faire quelque chose de suffisamment intéressant, vous devriez parler plutôt de tous les experts judiciaires. Il n'y a pas grand-chose à dire sur les interprètestraducteurs! » s'est exclamé un expert interprètetraducteur âgé de 64 ans, inscrit depuis 17 ans sur la liste d'une grande cour d'appel et sur la liste de la Cour de cassation, lorsque nous lui avons annoncé vouloir travailler sur cette spécialité d'experts. Tandis qu'une autre, âgée de 60 ans et inscrite depuis 13 ans sur la liste d'une petite cour d'appel, évoque même l'idée que "dans l'esprit des gens, ce n'est pas un métier. Et c'est aussi le cas dans l'esprit des policiers et des traducteurs eux-mêmes ».

\footnotetext{
14 «Le phénomène central de la vie professionnelle est le lien qu'une profession entretient avec son travail, un lien que j'appellerai "juridiction". Analyser le développement d'une profession revient à analyser la manière dont ce lien est créé dans le travail, dont il se matérialise dans des structures sociales formelles et informelles, et dont le jeu des liens de juridictions entre professions détermine l'histoire de chacune de ces professions » avance A. Abbott (1988) (cité par Le Bianic, 2008).
}

\section{LES FRAGILES INDICES D'UN PROCESSUS DE PROFESSIONNALISATION}

A contrario, plusieurs évolutions récentes témoignent d'une réalisation partielle de "l'idéal-type structuro-fonctionnaliste » du statut professionnel (Paradeise, 1988), lorsque ce dernier est appréhendé à travers des critères relatifs à la formation et à la socialisation professionnelle, aux compétences, à la dimension éthique ou d'auto-contrôle par les pairs. Bien que la définition canonique des professions qu'elle fournit exclue d'office l'activité ici considérée sur la base de son caractère accessoire, une dynamisation de cette perspective permet d'identifier un réel processus de professionnalisation des experts interprètes-traducteurs. Celui-ci apparaît toutefois bien complexe et surtout fragile.

\section{Formation et sentiment d'appartenance}

En matière de formation en effet, si l'hétérogénéité des cursus est a priori la règle puisqu'aucun diplôme n'est exigé pour candidater et être inscrit sur les listes, les interprètes-traducteurs ayant répondu au questionnaire présentent une homogénéité certaine : $69 \%$ déclarent un niveau bac +3 ou bac +4 , tandis que $83 \%$ ont déclaré des cursus en langue et $34 \%$ en droit (en France, à l'étranger, ou en France et à l'étranger). Un diplôme universitaire de deux années destiné à former des experts interprètes-traducteurs auprès de la justice s'est par ailleurs ouvert en 2005 à l'université de Nice. Enfin, c'est en matière de formation continue que l'évolution est la plus notable. L'obligation de formation des experts judiciaires a en effet été singulièrement renforcée par la législation de 2004, qui a posé comme critère objectif de réinscription sur les listes le suivi régulier de cycles de formation en lien avec la spécialité expertale ou l'expertise de justice. Et si seuls $58 \%$ des répondants au questionnaire ont indiqué suivre des formations en lien avec leur titre d'expert judiciaire (en moyenne une à deux fois par an), ce pourcentage atteint $70 \%$ parmi les experts interprètes-traducteurs «professionnels » et plus de $90 \%$ parmi les experts inscrits sur les listes de région parisienne (Paris, 
Versailles). Certes, l'essentiel de ces formations ne sont pas propres à la spécialité en langues, mais nul doute qu'elles contribuent à ce que - autre critère indispensable pour que se forme une profession - un peu plus de la moitié des experts interprètestraducteurs ayant répondu au questionnaire se sentent appartenir à la communauté des experts judiciaires ${ }^{15}$.

\section{L'existence d'associations professionnelles fragiles}

Un autre des critères classiques identifiés par la sociologie fonctionnaliste comme étant au fondement des professions porte sur l'existence d'associations professionnelles. Ces instances apparaissent comme des «leaders des mouvements professionnels» (Sarfatti, Larson, 1988) et à ce titre comme des vecteurs de professionnalisation. Répandues parmi les experts judiciaires, elles portent dans ce milieu le nom de compagnies. Encouragées par l'institution judiciaire qui y trouve des interlocuteurs avec qui discuter lors des réformes, et des relais pour diffuser le modèle d'un « bon expert » dessiné par les magistrats, ces compagnies locales ou nationales, pluridisciplinaires ou mono-disciplinaires, sont organisées dans une fédération nationale. Et comme l'analyse L. Dumoulin (2007), elles jouent un rôle de régulation qui s'apparente en partie à celui opéré par les ordres professionnels, tout en restant subordonnées à l'institution judiciaire et aux juges qui constituent les donneurs d'ordre intouchables de ce marché de l'expertise judiciaire. Les experts interprètestraducteurs, de ce point de vue, s'inscrivent bien dans ce schéma. Mais, une nouvelle fois, ils en sont quelque peu à la marge.

Certes, il existe bien une commission « expertise de justice » au sein de la Société française de traduction (SFT, principale association d'interprètes-traducteurs professionnels) et surtout deux compagnies monodisciplinaires, l'Union nationale des experts traducteurs et interprètes auprès des cours d'appel (UNETICA, crée en 1986) et la Compagnie des experts traducteurs et interprètes près la cour d'appel de Paris (CETICAP) ; mais la seconde a subi récemment une liquidation judiciaire et n'est qu'en voie de

${ }^{15}$ Contre $45 \%$ qui expriment le contraire et moins de $3 \%$ de nonréponses. renouveau tandis que seules deux compagnies monodisciplinaires locales ayant une activité au-delà d'un site internet non tenu à jour ont pu être recensées, à Paris et Aix-Marseille. En outre, même si $40 \%$ des répondants à notre questionnaire déclarent être membres d'une compagnie (quelle qu'elle soit), ces compagnies apparaissent bien plus comme des relais d'information que comme des représentants professionnels ${ }^{16}$. De fait, tous nos interlocuteurs - greffiers, magistrats, experts judiciaires représentants de compagnie, y compris d'interprètes-traducteurs - ont insisté sur l'atomisation, sinon l'absence, des instances de représentation des experts interprètestraducteurs, qui se concrétise par exemple par une grande difficulté à joindre de potentiels adhérents. "Le principal problème avec lequel on doit composer est leur dispersion à tous les niveaux. Il faut sans cesse leur courir après... », explique le responsable d'une compagnie pluridisciplinaire.

\section{Un rôle de revendication, de représentation, de socialisation}

Cependant, ces instances de professionnalisation remplissent un triple rôle : revendicatif, représentatif et de socialisation, passant respectivement par la formulation de doléances auprès des autorités judiciaires; par leur participation à la procédure d'inscription des candidats et de réinscription quinquennale des experts inscrits; et par l'organisation de cycles de formation. De ce point de vue, la loi de 2004 a fourni aux compagnies et à leurs dirigeants des ressources qui ont singulièrement renforcé ces deux dernières fonctions. La portée socialisatrice de ces instances passe ainsi, au-delà de la formation déjà évoquée, par leur rôle d'information quant au statut ou aux pratiques attendues par l'institution judiciaire. Les

16 Traitée sous forme de scores selon les différents items, une question portant sur les divers types de bénéfices retirés de l'appartenance à une compagnie fait nettement apparaître l'information (score de 1,06) comme un bénéfice plus important que la représentation syndicale ou la visibilité professionnelle (scores d'environ 0,20). Dans les questionnaires, des questions fermées multiples portaient sur les bénéfices tirés de l'appartenance à une instance représentative, distingués en trois catégories : informationnels, d'échanges et autres, chacune composée de plusieurs items. À chaque modalité de réponse assortie aux items - « un peu », « beaucoup », " assurément »- a été attribué le barème suivant : $0,2,0,3$ et 0,5 Sur cette base ont donc été calculés des scores permettant de situer le poids accordé à chaque bénéfice tiré de l'appartenance à une instance représentative. 
questions posées par les experts, lors des formations, vont en effet de «comment apposer son tampon sur les documents traduits? » à « que dois-je répondre à un prévenu qui me demande conseil ?». Quant à la fonction de représentation, elle a été officialisée en 2006 par la constitution d'un groupe de travail spécifique auprès de la Chancellerie, auquel sont associés des membres interprètes-traducteurs de compagnies mono ou pluridisciplinaires. Et les compagnies sont également appelées, depuis les textes de 2004, à participer au « recrutement» des experts en formulant un avis sur chaque dossier de candidature à l'inscription ou à la réinscription. Auparavant officieux - les magistrats en charge du service des expertises sollicitant telle ou telle personnalité -, ce rôle de représentation n'en est pas moins problématique, tant il s'entremêle, dans les faits, avec celui de revendications qui sont loin d'être unifiées. Des luttes internes complexes, liées notamment au titre d'expert judiciaire qui ouvre la possibilité d'entrer sur le marché des traductions assermentées réalisées pour des clients privés, constituent en effet de sérieux obstacles à la professionnalisation de l'activité d'expertise pour la justice.

\section{LES OBSTACLES À UNE PROFES- SIONNALISATION COMPLEXE}

Les actions menées par les instances représentatives constituent ainsi des baromètres situant le positionnement d'une partie des experts. Indépendamment de leur représentativité, elles reflètent une orientation donnée au type de professionnalisation revendiquée. De ce point de vue, le chantier des tarifs ainsi que les tentatives de fermeture d'un marché de la traduction auxquelles les actions de ces instances renvoient contribuent à complexifier le processus de professionnalisation attendue. Celle-ci n'en reste pas moins structurellement difficile, tant elle porte sur l'embô̂tement de deux marchés du travail, expliquant la complexité des luttes qui se déroulent entre experts.

\section{L'emboîtement inversé de deux marchés de l'interprétariat-traduction}

$\mathrm{Si}$ les principales revendications portent sur une revalorisation tarifaire des prestations linguistiques, dont le montant n'avait pas varié depuis 1999 et qui a été finalement obtenue en septembre 2008, la volonté de durcissement des conditions d'accès au titre d'expert structure en réalité fortement les actions des représentants des compagnies d'interprètes-traducteurs. Et ce pour une raison simple : le titre d'expert judiciaire ouvre l'accès à un marché judiciaire - celui des prestations pour le compte de la Justice - mais aussi et en réalité bien plus à un autre, cette fois juridique - celui des prestations assermentées, c'està-dire «certifiées conformes». Les enjeux de la détention du titre d'expert excèdent donc de loin le seul cadre judiciaire puisque qu'il habilite ses détenteurs à effectuer des prestations pour le compte d'autres commanditaires que les juridictions ou les commissariats de police et ce, toujours au titre de leur assermentation. Les prestations alors réalisées pour les seconds (particuliers, entreprises, avocats...) résultent de l'obligation d'apposer un sceau attestant d'une traduction assermentée pour un certain nombre de documents à destination des administrations publiques ${ }^{17}$. L'inscription sur une liste d'experts est donc un signal pour une potentielle clientèle privée indiquant, selon la formule de J.-Y. Trépos (1996), que «c'est l'État qui garantit indirectement que des experts privés ont un label auquel puissent croire des entreprises privées ». Autrement dit, et contrairement aux autres spécialités d'experts dont le statut peut se concevoir ou se vivre comme l'aboutissement d'une carrière ou un élément distinctif qui peut avoir des retombés positives sur leur clientèle et leur position sur le marché, pour les interprètes-traducteurs, c'est l'accès même au statut d'expert judiciaire qui conditionne l'entrée sur le marché des prestations assermentées. Un marché qui, de fait, est le plus important pour eux en termes de clientèles potentielles, de volumes d'activité et d'émoluments ${ }^{18}$. Cette imbrication entre deux marchés communs à tous les experts, ici renversée de manière singulière pour les interprètes-traducteurs, n'est pas sans consé-

\footnotetext{
${ }^{17}$ Comme dans le cas de procédure de naturalisation, de traduction de diplômes étrangers, de dossiers d'adoption dans un pays étranger, etc.

${ }^{18}$ La traduction de documents d'état civil, de diplômes ou autres actes authentiques est obligatoirement soumise à l'apposition d'un sceau expertal, ce qui réserve d'office certaines traductions aux experts traducteurs. Il en va de même concernant les prestations orales pour lesquelles certaines configurations (mariage...) nécessitent l'intervention d'un expert interprète.
} 
quences sur le type de professionnalisation possible et les obstacles que rencontre un tel processus.

\section{L'augmentation des tarifs: un double objectif}

Ainsi, les deux chantiers évoqués de revalorisation tarifaire et de durcissement des conditions d'accès au statut ne sont pas sans relation. En effet, comme le souligne, à propos des interprètes-traducteurs, un rapport de la Cour des comptes relatif aux frais de justice pénale en date du 23 mai 2006 :

«Les personnes auxquelles s'adressent les réquisitions participent au service public, cependant, le maintien de tels tarifs, parfois totalement irréalistes, que n'a pas contesté la direction du budget, ne favorise pas l'appel aux prestataires les plus qualifiés, qui sont en droit d'exiger une juste rémunération de leur intervention. Il peut même constituer une incitation à la fraude, laquelle peut porter sur le nombre, sur la nature ou sur la durée des actes. »

Ce constat objectif se traduit par des cris du cœur" je suis payé autant qu'une femme de ménage, voire moins, alors que j'ai un doctorat" s'indigne un interprète interrogé au cours d'une séance de formation - et des opinions massivement orientées : plus de $70 \%$ des répondants aux questionnaires considèrent qu'une revalorisation des tarifs serait facteur pour eux de reconnaissance, et de légitimité accrue. De fait, comme l'indique une experte dont la traduction est l'activité principale, «les tarifs ne sont pas le reflet de la profession ", tout l'enjeu étant ici ce que recouvre ce terme de "profession». En ce sens, il apparaît que les revendications pécuniaires visent autant à améliorer les conditions de travail et le prestige symbolique des experts interprètestraducteurs qu'à fermer pratiquement un second marché professionnel qui dépasse, de loin, les seules demandes effectuées par l'institution judiciaire.

\section{Fermer les conditions d'accès au titre?}

En effet, un pan important des revendications des compagnies porte sur la nature de l'activité professionnelle principale. Le titre d'expert interprètetraducteur est ainsi perçu comme étant nécessaire- ment lié au fait d'exercer comme activité professionnelle principale la traduction et/ou l'interprétariat, idéalement en libéral, le critère formel résidant dans le paiement de cotisations à l'URSSAF (Union de recouvrement des cotisations de sécurité sociale et d'allocations familiales). Ce critère d'accès pratique est même érigé en condition indispensable pour pouvoir adhérer à ces instances représentatives, comme l'explicitent leurs sites internet. Pour adhérer à la compagnie parisienne (CETICAP) par exemple, il faut envoyer un chèque de 100 euros, "accompagné de la photocopie de votre attestation URSSAF 2008, ou de tout autre document permettant de justifier de cette affiliation ».

Pour ces instances, les experts judiciaires «non professionnels »-c'est-à-dire non soumis à ces cotisations URSSAF - « cassent les prix sur le secteur d'activité en acceptant tout: tant en terme d'horaires qu'en terme de prix » détaille une interprète membre de la compagnie nationale, interrogée au cours d'une séance de formation. En effet, si les missions judiciaires sont cantonnées dans des tarifs légalement fixés, ce n'est pas le cas des prestations effectuées en tant qu'expert judiciaire pour le compte de clients autres que la Justice, pour lesquelles les prix sont fixés de manière discrétionnaire par le traducteur expert ${ }^{19}$. Le positionnement des instances se traduit par des demandes formulées explicitement à la Chancellerie, mais également par la manière dont les membres de ces instances participent au processus d'inscription des candidats et de réinscription des experts par les cours d'appel. Le paiement de cotisations à l'URSSAF est ainsi explicitement formulé dans les avis apposés sur ces candidatures, comme nous l'avons constaté dans les deux cours d'appel où nous avons pu étudier des dossiers. Une interprète-traductrice anciennement adhérente d'une instance - car en désaccord avec ces revendications de clôture du marché et parlant de "véritable chasse aux sorcières $»-$ indique ainsi :

«Elles [instances représentatives] organisent des doubles listes d'experts : ceux qui sont "en règle" car détenant un numéro d'URSSAF et les autres (...).

\footnotetext{
${ }^{19}$ C'est le cas, par exemple, de traduction de diplôme lors d'une inscription dans un établissement d'enseignement français ou de documents d'état civil dont la traduction en français par un traducteur
} expert incombe à la personne souhaitant faire valoir ces documents. 
Il y a l'idée de traquer les confrères irréguliers, ce qui est une dérive puisque ce ne sont pas des organes disciplinaires. »

Ainsi la pratique revendicative des membres de ces instances prend une autre tournure : il ne s'agit plus de représenter tous les experts interprètes-traducteurs, mais bien de peser sur la réglementation du statut de ces experts, en présentant l'une de ces figures d'expert comme étant la seule légitime à détenir le titre.

\section{Restreindre l'accès au double marché ou l'enjeu de la crédibilité professionnelle}

Une telle représentation pourrait laisser supposer que ces revendications formalisent un affrontement entre experts « professionnels » et « experts non professionnels ». Pourtant, elle occulterait le fait que les membres des compagnies ne sont pas exclusivement interprètestraducteurs professionnels. Outre la forte proportion des « non-professionnels » membres d'instances représentatives $-46 \%$ parmi les répondants au questionnaire - leur présence, au cours de séance de formations pour experts judiciaires, va dans le même sens. En réalité, le critère et la lutte professionnels/non professionnels sont transcendés par deux dimensions: la qualité des traductions d'une part, et la disponibilité des experts d'autre part. Force est de constater alors que chacun des acteurs - les interprètes " professionnels », « non professionnels », mais aussi la Chancellerie et les juges - ne met pas l'accent de manière égale sur ces deux dimensions. La qualité des prestations est en effet une revendication des experts «professionnels», bien plus que des magistrats qui, à l'inverse, privilégient la disponibilité des experts comme dimension essentielle à réguler ${ }^{20}$. On aurait pu pourtant s'attendre à exactement l'inverse - les experts revendiquant une régulation de leur disponibilité, et les magistrats cherchant à s'assurer de la qualité des prestations de traduction. Mais l'État semble défendre un enjeu bien plus procédural que substantiel : assurer la réalisation des opérations de traduction plus que d'en vérifier la qualité.

\footnotetext{
${ }^{20}$ Les dossiers d'experts auxquels nous avons eu accès le confirment : on y constate la présence de fiches signalétiques remplies par les services de police rendant compte du nombre de refus d'intervention par un expert et des motifs apportés à ceux-ci, qui côtoie, à l'inverse, des lettres de recommandations de magistrat vantant la disponibilité de l'expert.
}

Car c'est bien la qualité des prestations qui est essentielle aux yeux des responsables de compagnies d'interprètes : "il y a trop de personnes incompétentes assermentées, ce qui est associé au fait que les tarifs font fuir les personnes compétentes à terme... » analyse l'un d'eux. Certains ont même été jusqu'à fournir l'identité d'experts parlant mal ou très mal le français pour illustrer leurs dires; et l'une des compagnies revendique auprès de la Chancellerie l'établissement d'un examen linguistique... en français! L'enjeu résiderait donc davantage dans la volonté de délimiter les contours d'un marché professionnel composé de prestataires de services " crédibles» que dans celle d'en homogénéiser le contenu aux seuls membres en exercice libéral. C'est finalement le lien avec la sphère des langues qui constitue le critère fédérateur selon ces instances (et en ce sens la crédibilité et la capacité à envoyer à l'extérieur une image de bon professionnel), plutôt que le fait d'avoir un statut spécifique. Les formateurs et enseignants en langue, contrairement à d'autres, ne sont ainsi pas exclus des compagnies ni stigmatisés lors des procédures d'inscription ou de réinscription sur les listes.

Encadré 4

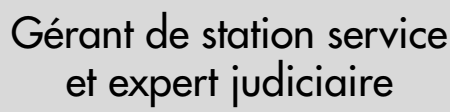

Un expert interprète-traducteur ayant des responsabilités dans une compagnie indique que l'envoi d'une pétition aux membres de la compagnie visant à appuyer leur demandes de relèvement de tarifs auprès des autorités illustre à elle seule l'hétérogénéité problématique des experts, certains n'y étant manifestement " pas à leur place ». À titre d'exemple, il évoque la signature apposée d'un expert dont le tampon mentionnait ses fonctions de gérant d'une station service. Cet exemple, non anecdotique selon lui, illustre les « cumuls invraisemblables d'activités professionnelles qui décrédibilisent les experts interprètestraducteurs et contribuent à l'image selon laquelle ce n'est pas un métier ». 
PROFESSIONNALISATION DES EXPERTS : LE DOUBLE JEU DE L'ÉTAT

Il est pourtant étonnant de constater la faiblesse des revendications des experts à propos de la disponibilité attendue par les magistrats. C'est bien là, en effet, que se noue selon nous un autre enjeu au fondement de la dimension problématique du processus de professionnalisation mis en évidence.

\section{Listes cour d'appel \\ et listes « du procureur »}

L'enjeu de la disponibilité des experts conduit ainsi l'État, ou plus exactement les magistrats, à jouer un double jeu à l'égard des compagnies. Au-delà, en effet, de la Chancellerie dont l'activité, au service des experts, vise à sélectionner de «bons experts", compétents et reconnus, et qui pourrait être sensible à la stratégie menée par les instances représentatives, les magistrats rencontrent des contraintes et donc disposent d'une liberté qui leur permet au quotidien de saper ce processus de professionnalisation des experts judiciaires interprètes-traducteurs. Il suffit en effet, comme pour tout expert judiciaire, de faire prêter serment à n'importe qui pour le transformer en interprète «procéduralement» valide aux yeux de la justice. Cette liberté, inscrite dans les textes et à laquelle les magistrats recourent peu pour d'autres spécialités (comptables et psychiatres par exemple, mais aussi ingénieurs ou experts en art) est ainsi massivement employée, en pratique, par les juges et les autres acteurs qui ont besoin d'interprètestraducteurs. «Les juridictions et les services de police ont souvent recours à beaucoup de personnes "de proximité" pour palier le manque et parfois la disponibilité manquante des experts, ce qui fait que certains [prestataires] font le siège à la police pour bénéficier de prestations à effectuer » déclare une experte interrogée au cours d'une séance de formation.

De fait, ces pratiques s'incarnent dans des dispositifs et des outils créés par les magistrats pour faire face à cette indisponibilité récurrente, selon eux, des experts interprètes-traducteurs. Nous avons ainsi découvert des listes parallèles en interrogeant des magistrats ; appelées « les listes du procureur », elles sont même officiellement reconnues depuis un décret de mars 2005 en application de la loi de 2004 sur les experts. "Les juges utilisent plutôt la liste des cours d'appel ou de la Cour de cassation, mais la police des frontières par exemple, à la préfecture, et un grand nombre de commissariats n'utilisent que cette liste $d u$ procureur en fait. C'est très rare qu'ils viennent piocher dans la liste cour d'appel. Alors c'est vrai que la compagnie râle, je les comprends, mais faut-il pour autant un document unique, avec uniquement les experts cour d'appel et les autres mélangés, ou deux listes? Moi, ici, j'ai résolu d'avoir deux listes et surtout, j'ai diffusé ces deux listes partout» indique un magistrat en charge du service des expertises dans une cour d'appel de province.

Ces pratiques se justifient par le manque de disponibilité des experts «officiels », ceux qui sont inscrits sur la liste établie par chaque cours d'appel. C'est "le refus de certains experts d'exercer à de tels tarifs [qui] conduit les autorités à avoir recours à cette main-d'œuvre de secours » explique une interprète, cette «main-d'œuvre» étant bien sûr jugée de moindre qualité que celle que les magistrats peuvent trouver dans les listes cours d'appel.

\section{Des listes contournées dans la pratique}

De fait, ces nouveaux acteurs de proximité sur le marché judiciaire constituent des concurrents supplémentaires, qui ont échappé au filtre de l'inscription sur les listes et représentent des obstacles au processus de professionnalisation porté par les compagnies. Et si leur qualité est critiquée, leur disponibilité les préserve de toute exclusion durable d'un jeu où ce sont toujours les magistrats qui orientent, structurent et ouvrent - ou non - un marché ouvert et atomisé, quasi impossible à clore. En effet, qu'elles soient officielles ou dites «du procureur», c'est l'existence même de listes qui semble ne pas s'avérer nécessaire dans les pratiques développées par les magistrats.

La pratique des juges conduit donc à apprécier de manière extensive le recours à un interprète dans les cas où un prévenu n'est pas francophone, l'impératif de tenue des audiences et de satisfaction immédiate des besoins de traduction primant sur celui de choix 


\section{Encadré 5 \\ Une observation au tribunal correctionnel : comment l'enquêtrice aurait pu se retrouver interprète}

Au cours d'une audience au tribunal correctionnel, le juge doute de la capacité du prévenu, d'origine étrangère, à comprendre ce qui lui est expliqué en français. Aucun interprète n'ayant été réquisitionné, cela pose un problème pour la tenue de l'audience. Étant la seule dans la salle d'audience, je suis interpellée: "Vous accompagnez M. B. ? 》. Suite à ma réponse négative, et avant de décider ou non de procéder au report de l'audience, le juge demande au greffier si le prévenu est venu seul afin de demander à un éventuel accompagnant de lui traduire les propos tenus laprès avoir demandé au prévenu si quelqu'un ne pouvait pas l'assister pour traduire les propos, sans que ce dernier ne comprenne la question qui lui était posée). Finalement, le greffier est allé chercher un proche du prévenu attendant à l'extérieur de la salle d'audience afin que ce dernier prête son concours en traduisant les propos du prévenu et du magistrat.

de l'interprète cantonné aux seules listes d'experts judiciaires ${ }^{21}$.

L'article D506 du Code de Procédure Pénale prolonge d'ailleurs cette acception du recours à l'interprète en indiquant qu'il ne doit être fait appel à ce dernier qu'en cas de "nécessité absolue » soit « si le détenu ne parle ou ne comprend la langue française et si il ne se trouve sur place aucune personne capable d'assurer la traduction ». Les dispositions réglementaires institutionnalisent donc une forme de dimension supplétive voire dérogatoire du recours aux experts interprètes en écartant le risque pour une procédure d'être entachée de nullité en cas d'absence d'intermédiaire en langue. C'est pourquoi le «processus réel de professionnalisa-

\footnotetext{
${ }^{21}$ Sur l'importance de ces impératifs pratiques dans le déroulement des audiences et le fonctionnement quotidien de la justice, voir, à propos cette fois de dispositifs techniques comme la visioconférence, C. Licoppe et L. Dumoulin (2007).
}

tion des experts judiciaires », établi par L. Dumoulin (2007) de manière générale, induit par une impulsion commune permise par "une convergence d'intérêts » des magistrats et une partie des experts judiciaires, semble rencontrer de sérieux et spécifiques obstacles pour les experts interprètes-traducteurs. Les constats faits à leur sujet incitent plutôt à adopter une lecture singulière pour cette spécialité du fait des orientations contradictoires impulsées par la législation en matière d'expertise: le renforcement d'une forme de cogestion entre compagnies et chancellerie, s'inscrivant dans un mouvement de professionnalisation, se double en effet des pratiques des magistrats en matière de nomination d'experts interprètes-traducteurs, qui contournent fréquemment les listes lorsqu'ils ont recours à ces prestataires de services en langues, minant par là même ce processus de professionnalisation des experts.

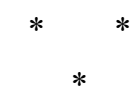

Le point de départ de l'étude résidait dans la focalisation sur une catégorie professionnelle ouvrant des perspectives d'approches différenciées : une activité de travail impliquant le support linguistique, une immixtion dans la sphère judiciaire, une relative indétermination statutaire, l'appréhension d'un groupe morcelé et marginalisé, l'appréciation d'un exercice expertal spécifique. L'expertise judiciaire permet d'introduire une posture hybride à bien des égards : entre deux activités professionnelles (principale et accessoire), entre un statut expertal et un statut professionnel, entre une posture profane et une posture spécialisée. C'est une des caractéristiques rendant peu aisée toute démarche didactique et qui explique, au moins partiellement, le chevauchement d'injonctions parfois contradictoires auxquels les experts interprètes-traducteurs sont soumis : à la fois experts judiciaires, prestataires (même de manière ponctuelle) de services en langues, garants d'un droit fondamental, et éventuellement professionnels d'un autre domaine d'activité. Plutôt que de considérer ces appartenances différenciées comme des entraves, la présente démarche tend à les appréhender comme des composantes d'un processus de professionnalisation. Celui-ci semble toutefois, au final, structurellement problématique, sinon impossible. 
Les experts interprètes-traducteurs apparaissent en effet comme des collaborateurs de la justice dans une posture ingrate: marginalisés par rapport au corps expertal en raison de caractéristiques inhérentes à la composition du groupe mais aussi renvoyant à la nature de l'activité qu'ils sont amenés à réaliser. Les indices de professionnalisation évoqués illustrent l'amorce d'un processus effectif mais pas pour autant univoque. L'existence de revues spécialisées, d'instances représentatives, d'un groupe de réflexion auprès des autorités, de parcours de formation, mais également d'un sentiment d'appartenance commun pour une partie d'entre eux - qui recourent au terme de métier ou de profession pour désigner leur statut d'expert interprète-traducteur - tend à illustrer un certain degré de cohésion. Mais la volonté du législa- teur de se garder de l'immixtion de l'expert judiciaire dans la procédure pénale et de maintenir une liberté totale pour le magistrat dans le choix du recours à un expert, et à quel expert, constitue, à coup sûr, un frein à ce processus de professionnalisation; processus qu'il encourage par ailleurs pour améliorer la qualité des prestations qu'il attend de ces experts. L'embồtement des marchés professionnels et des activités à destination judiciaire et à destination juridique accentue encore la complexité du processus, tout en aiguisant les enjeux, en particulier pour les interprètes-traducteurs professionnels ; ces derniers étant soumis à une entrée sur un marché peu rémunérateur, peu valorisé et très contraignant pour accéder à un marché bien plus conséquent, divers, où ils peuvent véritablement bénéficier de leur statut d'indépendant.

\section{Bibliographie}

Abbott A. (1988), The system of professions: an essay on the division of expert labor, Chicago University Press.

Bessy C. et Chateaureynaud F. (1995), Experts et faussaires. Pour une sociologie de la perception, Métaillié.

Bourdieu P. (1982), Ce que parler veut dire. L'économie des échanges linguistiques, Paris, Fayard.

Chapoulie J.-M. (1973), « Sur l'analyse sociologique des groupes professionnels », Revue française de sociologie, vol 14, $\mathrm{n}^{\circ} 1$, pp. 86-114.

Chauvaud F. (2003) (avec la collaboration de L. Dumoulin), Experts et expertise judicaire, France $X I X^{e}$ et XXe siècles, Rennes, Presses universitaires de Rennes.

Dumoulin L. (2007), « Les experts judicaires ont-ils encore du pouvoir? Des effets de la professionnalisation des experts sur la Justice», in E. Rude-
Antoine (dir.), Le procès enjeu de droit, enjeu de vérité, Paris, PUF.

Dumoulin L. (2007), L'expert dans la Justice. De la genèse d'une figure à ses usages, Economica, Coll. «Études politiques».

Dumoulin L., Licoppe C. (2007), «L'ouverture des procès à distance par visioconférence: activité, performativité, technologie $»$, Réseaux $\mathrm{n}^{\circ} 25$.

Harvey M. (2005), «Incroyable mais vraie: la traduction juridique », in R. Greenstein (dir.), $L a$ langue, le discours et la culture en anglais du droit, Paris, Publications de la Sorbonne, pp. 57-68.

Heinich I. (1984), « Les traducteurs littéraires : l'art et la profession ", Revue française de sociologie, vol. $25, \mathrm{n}^{\circ} 2$, pp. $264-280$.

Gemar J.-C. (1987), « La traduction juridique : art ou technique d'interprétation », Ottawa, Revue générale de droit. 
Karpik L. (1996), Les avocats. Entre l'État et le marché, Paris, Gallimard.

Lascoumes P. (2002), «L'expertise, de la recherche d'une action rationnelle à la démocratisation des connaissances et des choix», Revue française d'administration publique 2002/3, $\mathrm{n}^{\circ} 103$, pp. 69377.

Le Bianic T., Vion A. (2008), Action publique et légitimité professionnelle, LGDJ, coll. « Droit et Société ».

Mathieu Fritz A. (2005), Les huissiers de justice, Paris, PUF, coll. « Sciences sociales et société ».

Trepos J-Y. (1996), La sociologie de l'expertise, Paris, PUF, coll. « Que sais-je ? », nº 3119.

Paradeise C. (1988), "Les professions comme marchés du travail fermés ", Sociologie et sociétés, vol. $20, \mathrm{n}^{\circ} 2$, pp. $9-21$.
Radimsky J. (2004), «Dans quelle mesure est-il possible de traduire un terme juridique ? », Document de travail L25, Brnenske Univerzity.

Roussel V. (2007), «Les changements d'ethos des magistrats », in Commaille J. et Kaluzynski (dir.), $L a$ fonction politique de la justice, Paris, La Découverte, coll. « Recherche».

Sapiro G. (2008), Translation. Le marché de la traduction en France à l'heure de la mondialisation, Paris, CNRS Éditions.

Sarfatti Larson M. (1988), «A propos des professionnels et des experts ou comme il est peu utile d'essayer de tout dire", Sociologie et Sociétés, vol. 20 , pp. $23-40$.

Terre F. (1986), «Brèves notes sur les problèmes de la traduction juridique » Revue internationale de droit comparé, vol. $38 \mathrm{n}^{\circ}$ 2, pp. 347-350

\section{Résumé}

\section{Une professionnalisation problématique : les experts judiciaires interprètes-traducteurs \\ Keltoume Larchet, Jérôme Pélisse}

Centré sur l'analyse du statut et des pratiques des interprètes-traducteurs œeuvrant pour le compte de la justice, cet article étudie un processus problématique de professionnalisation concernant cette activité. Les experts interprètes-traducteurs possèdent en effet un statut ambigu, commun aux autres experts judiciaires, mais aussi en grande part spécifique. Spécialistes en langue, ils peuvent en effet ne pas en être des professionnels reconnus. Au-delà, leurs caractéristiques sociodémographiques, la nature de leurs activités ou leurs conditions de travail en font des experts marginaux à l'activité invisible et dévalorisée. L'article se propose donc, en s'appuyant sur divers types de données, de comprendre le caractère problématique de cette professionnalisation et le rôle qu'y jouent les magistrats, clés d'entrée pour un double marché professionnel qui dépasse la seule activité d'expertise de justice.

Mots clés

Professionnalisation, interprète, traducteur, justice

Journal of Economic Litterature: I 44 